\title{
Tecnología móvil en el ambiente académico y su uso en videojuegos
}

\section{Mobile technology in the academic environment and its use in video games}

\author{
SOTO-HERNÁNDEZ, Ana María†, VARGAS-PÉREZ, Laura Silvia*, PERALTA-ESCOBAR, Jorge y \\ SALDAÑA-GARCÍA, Sergio
}

Instituto Tecnológico de Ciudad Madero / Tecnológico Nacional de México

ID $1^{\text {er }}$ Autor: Ana María, Soto Hernández / ORC ID: 0002-8660-3413, Researcher ID Thomson: X-2282-2018, CVU CONACYT ID: 317457

ID $1^{\text {er }}$ Coautor: Laura Silvia, Vargas Pérez / ORC ID: 0001-7605-9779, Researcher ID Thomson: X-2426-2018, CVU CONACYT ID: 212197

ID $2^{\text {do }}$ Coautor: Jorge, Peralta Escobar

ID $3^{\text {er }}$ Coautor: Sergio, Saldaña García

DOI: $10.35429 / \mathrm{JCS} .2019 .10 .3 .25 .37$

Recibido: 20 de Agosto, 2019; Aceptado 30 de Diciembre, 2019

\begin{abstract}
Resumen
El uso de los dispositivos móviles entre la población joven se ha generalizado, para leer periódicos, libros o revistas, buscar información para tareas escolares, y comunicarse con sus compañeros para actividades estudiantiles, lo cual puede utilizarse para motivar un mejor desempeño académico. Las nuevas aplicaciones y plataformas para la gestión del aprendizaje, relacionados con las nuevas tecnologías, cada vez más accesibles como la móvil, con teléfonos inteligentes o tabletas en el aula, obliga a los profesores, a utilizar esas ventajas en sus estrategias de enseñanza y aprendizaje. En este trabajo se presenta un estudio, en el Tecnológico Nacional de México, sobre cómo el uso de esta tecnología móvil está correlacionada con mejores resultados en el desempeño académico para estudiantes de ingeniería en matemáticas y del lenguaje escrito, no así para física. Así también de cómo el uso extendido de las redes sociales y de las aplicaciones para el ocio, como los videojuegos, puede convertirse en un elemento estratégico motivacional para el aprendizaje. El estudio también muestra la necesidad de fortalecer el compromiso de los profesores por vincular estas tecnologías efectivamente en el aula, y no dejarlo en una intencionalidad.
\end{abstract}

Dispositivos móviles, Estudiantes de ingeniería, Videojuegos

\begin{abstract}
The use of mobile devices among the young population has become widespread, to read newspapers, books or magazines, find information for school assignments, and communicate with their peers for student activities, which can be used to motivate better academic performance. New applications and platforms for learning management, related to new technologies, increasingly accessible such as mobile, with smartphones or tablets in the classroom, forces teachers to use these advantages in their teaching and learning strategies. This paper presents a study, at the National Technological Institute of Mexico, on how the use of this mobile technology is correlated with better results in academic performance for engineering students in mathematics and written language, not so for physics. So also, how the widespread use of social networks and applications for leisure, such as video games, can become a strategic motivational element for learning. The study also shows the need to strengthen the commitment of teachers to link these technologies effectively in the classroom, and not leave it in an intentionality.
\end{abstract}

Mobile devices, Engineering students, Video games

Citación: SOTO-HERNÁNDEZ, Ana María, VARGAS-PÉREZ, Laura Silvia, PERALTA-ESCOBAR, Jorge y SALDAÑAGARCÍA, Sergio. Tecnología móvil en el ambiente académico y su uso en videojuegos. Revista de Simulación Computacional. 2019. 3-10: 25-37

\footnotetext{
* Correspondencia al Autor: (laura.silvia.vargas@gmail.com)

$\dagger$ Investigador contribuyendo como primer autor.
} 


\section{Introducción}

El uso extensivo de los dispositivos móviles entre la población, particularmente los jóvenes, puede utilizarse como recurso para motivar un mejor desempeño académico, que los profesores agradecerían. Cuando se conjunta el interés de los estudiantes y de los profesores por utilizar aplicaciones móviles con el fin de mejorar los aprendizajes que requieren los aspirantes a ingenieros, se tiene un doble beneficio.

Dentro de todos los intereses de los estudiantes al utilizar una aplicación móvil, el videojuego es el más atractivo, por lo que utilizarlo para fines educacionales, puede beneficiar el proceso de aprendizaje. Estas aplicaciones se ejecutan gracias a un programa de software-que es el videojuego propiamente dicho- procesado por una máquina que cuenta con dispositivos de entrada y de salida. Los distintos tipos de dispositivo en los que se ejecutan los videojuegos se conocen como plataformas, siendo las más populares: las computadoras, las videoconsolas, los dispositivos portátiles y las máquinas Arcade.

En las universidades, los profesores responsables de los cursos en los planes de estudio de ingeniería, especialmente los de mayor edad, han sido impulsados para actualizar sus estrategias didácticas basadas en el pizarrón y el gis, los apuntes y cuestionarios, los ejercicios en papel y las prácticas en el laboratorio con equipo experimental. No obstante, no ha sido muy exitoso el intento para que vean, estudien, analicen, evalúen y apliquen otra gran variedad de recursos didácticos y de plataformas para la gestión del aprendizaje, relacionados con las nuevas tecnologías.

Hoy en día, el uso del proyector conectado a una computadora se convirtió en un nuevo pizarrón donde los profesores tienen que mostrar sus habilidades para utilizar simuladores, programas diversos como aplicaciones que permiten tener al alcance de un clic el trazo de una gráfica, el despliegue de la trayectoria de un objeto, la evolución de una reacción química. Lo anterior, aunado al abaratamiento de las tecnologías móviles que permiten ver un gran número de estudiantes con teléfonos inteligentes o tabletas o computadoras personales en la propia aula, obliga a utilizar esas ventajas en las estrategias de enseñanza y aprendizaje por todos los profesores.
Sin embargo, a nivel institucional, sistémico, pareciera que "la escuela se está quedando al margen de esta transformación" (Pedró, 2016, pág. 21), porque las iniciativas se han desplegado de forma personal, los proyectos se han asumido casi como retos propios de los profesores, porque otros utilizan la tecnología en el ámbito personal pero apenas están incorporándola en su actividad docente, a pesar de "la casi universalización de los dispositivos inteligentes entre los alumnos y los docentes" (Pedró, 2016, p. 22). Y tratándose de los programas de ingeniería en México, 6.7\% usan las redes sociales como medio (Castro Romero, 2014).

El uso de los dispositivos electrónicos donde las aplicaciones de software están al alcance, se ha generalizado con la intención de aprovechar las tendencias de los jóvenes a estar conectados permanentemente de forma virtual. Por ello, el uso de las tecnologías de la información y la comunicación (TIC) se ha convertido en un eje transversal de la política educativa en todos los niveles como reflejo de la tendencia mundial que también ha sido objeto de estudio (OECD, 2015; Chiappe, 2016).

Sin embargo, pareciera que utilizar este tipo de recursos no ha motivado el mejoramiento de las competencias de los estudiantes en México, pues resultados de pruebas estandarizadas como Pisa y los cuestionarios para profesores plasmados en la encuesta denominada Talis, ambos de la OECD, así lo muestran (OECD, 2015; OECD, 2016; OECD, 2017).

Aún más, a nivel internacional se ha mostrado que "la calidad de los resultados en educación no tiene tanto que ver con la presencia o la ausencia de tecnología en las escuelas como con la pedagogía adoptada y las condiciones en que se aplica en el aula (Pedró, 2016, pág. 22). Dentro de la diversidad de recursos tecnológicos, los que más se han popularizado son los que tienen características lúdicas y se han asociado a los juegos entre niños y jóvenes.

Muchos de esos recursos -aplicacionesse han diseñado incorporando elementos para aprendizajes en varias disciplinas -aprendizaje basado en juegos, GBL por sus siglas en inglésprecisamente por sus características motivacionales, afectivas, colaborativas $\mathrm{y}$ sociales. 
Estas aplicaciones utilizan ambientes atractivos como las vistas 2D y 3D, la Realidad Aumentada (AR), con las versiones para dispositivos móviles como teléfonos celulares y tabletas (Bacca, y otros, 2015; Chen, Ho, y Lin, 2015; Diaz, Hincapié, y Moreno, 2015; Nincarean, y otros, 2013; Padilla, y otros, 2012; Samaniego, y Sarango, 2016; Sannikov, y otros, 2015). Además de ello, es muy conveniente aprovechar también la inclinación de jóvenes estudiantes de ingeniería en sistemas computacionales por la programación de aplicaciones para dispositivos móviles.

Esto es, no solamente el uso de aplicaciones desarrolladas para distintos fines es motivante en los estudiantes de las asignaturas básicas, la incorporación de estudiantes en el desarrollo de programas para construir aplicaciones aplicadas para el diseño de un videojuego, utilizando recursos como la Realidad Virtual y teniendo a la disposición equipos como las gafas apropiadas para ello, se convierte en un gran impulsor de las vocaciones para la programación. Sin embargo, aún con todo lo anterior, todavía pareciera que los profesores consideran que el uso de los dispositivos móviles dentro del aula por parte del estudiante es un distractor en el aprendizaje y debe ser proscrito en ese espacio, como ha sido planteado en diversos países.

Más allá de lo anterior, un sector de esos profesores sí tiene algunas estrategias, al menos de comunicación, a través de las redes sociales más comunes como Facebook y WhatsApp, que también han sido estudiadas y catalogadas como potencialmente positivas en el aprendizaje de acuerdo con diversos investigadores (Hershkovizt y Forkosh-Baruch, 2017; Gómezdel-Castillo, 2017; Ahern, Feller, y Nagle, 2016; Amry, 2014). Inclusive, los estudiosos han generado una categorización de acuerdo con el uso de las mismas por parte de los estudiantes (Gavilan, Martínez-Navarro, y Fernández-Lores, 2017).

No obstante, otro grupo de profesores trabaja con plataformas educativas que permiten institucionalizar la gestión del aprendizaje y unifican los esfuerzos en beneficio de las comunidades, la más conocida de las cuales, por su acceso libre, es Moodle, aunque también Schoology y Claroline tienen sus versiones libres, por no mencionar a las que requieren licencias como Blackboard.
Por ser la más extendida, Moodle ha sido estudiada más consistentemente (Rodríguez y Rivadulla, 2015) pero siempre requiere de una capacitación constante de los profesores para mantener el contacto y la motivación de los estudiantes (Hobbs y Tuzel, 2017; Dahlstrom, 2015) porque implican el desarrollo de recursos didácticos especializados utilizados en la modalidad virtual, principalmente.

En este siglo XXI, cuando el uso de los teléfonos inteligentes está creciendo exponencialmente de tal manera que se proyecta que para el 2020 el 70\% de la población mundial tendrá uno a su alcance (Ericsson, 2017), la utilización de esta tecnología para beneficios en la educación de las personas es una gran oportunidad.

Aunque no se debe perder de vista que aquellos estudiantes que se encuentran en el $30 \%$ restante deben contar con una alternativa real y cercana para no ser objetos de exclusión y falta de equidad. En las instituciones de educación superior, el acceso generalizado de los estudiantes a salas de cómputo brinda estas opciones indispensables.

Lo anterior implica revisar las competencias profesionales de los profesores y la infraestructura institucional disponible para que utilice las herramientas tecnologías, así sean equipos de cómputo y dispositivos móviles, programas o aplicaciones y la conectividad.

Los análisis y evaluaciones sobre el uso de las Tic en los sistemas educativos indican que es importante el carácter estructural de la política aplicada en ese sentido, mediante lo que Brandao y Vargas han llamado Trípode para la apropiación de tecnologías digitales en las escuelas, la cual está integrada por: la dimensión de la infraestructura física, con equipos, software, y los servicios necesarios para garantizar su funcionamiento; la dimensión técnica relacionada con la capacidad de estudiantes, profesores y directivos para llevar a cabo los proyectos o intervenciones, y la dimensión política vinculada con los acuerdos y compromisos por todos los actores institucionales para darle sentido a los proyectos e intervenciones propuestas (2016, págs. 10-11). 
Lo anterior está vinculado con el estudio de las cualidades del estudiante y del profesor, de su contexto, y el uso de las tecnologías de la información y la comunicación, y los dispositivos móviles. Lo antes dicho ha conducido a las preguntas de investigación que motivan este documento ¿Cómo utilizan los estudiantes los dispositivos móviles que tienen al alcance de la mano? ¿Los utilizan para fines educacionales? ¿Qué beneficios tienen en sus habilidades y conocimientos básicos para una carrera de ingeniería? ¿Cómo los utilizan los profesores de ingeniería en su trabajo con los estudiantes?

Las preguntas se han investigado a partir del análisis estadístico de las bases de datos de los resultados de los estudiantes en sus exámenes de admisión al instituto ITCM durante el 2016, y de las respuestas hacia algunas preguntas del cuestionario de contexto del examen EXANI-II del Centro Nacional para la Evaluación de la Educación Superior (CENEVAL).

Así también, se utilizaron para esta investigación los resultados de un estudio sobre el uso de los dispositivos móviles entre la población estudiantil del ITCM realizado en el 2017 (Santiago, Pérez, y Soto, 2017) que proporciona información también sobre el uso que los profesores hacen de él.

Finalmente, también se consideraron los resultados de un estudio sobre competencias digitales, informacionales y pedagógicas de los profesores y sus actitudes hacia el uso de las Tic, realizado en el 2017 (Saldaña, 2017).

\section{Desarrollo}

Este trabajo forma parte de una investigación con estudiantes de nuevo ingreso en programas de ingeniería en el sur del estado de Tamaulipas, México. Después del proceso de ingreso al ITCM, para el segundo semestre de 2016, se analizaron los resultados de 1581 sustentantes del examen estandarizado EXANI-II Admisión del CENEVAL, referentes a las habilidades de Pensamiento Matemático y de Pensamiento Analítico, de Estructura de la Lengua y de Comprensión Lectora.

De la misma manera se analizaron los resultados para el Módulo de Ingeniería del EXANI-II, constituido por las áreas de Matemáticas, Física, Lenguaje Escrito e Inglés.
Se analizaron las respuestas a las preguntas del Cuestionario de Contexto del EXANI-II que incluían el uso de los dispositivos móviles para: leer periódicos, libros o revistas, o como ayuda para buscar información por tareas escolares, o como un medio de comunicación con sus compañeros de clase para los mismos fines.

El análisis se realizó para indagar la posible correlación con los resultados de los exámenes sobre las habilidades y de los conocimientos, plasmados en los módulos de Admisión e Ingeniería, respectivamente.

Así mismo, estos indicadores sobre los dispositivos móviles se vincularon con indicadores como el género del sustentante, la modalidad y el régimen del bachillerato de procedencia, y la carrera a la que aspiraban.

Por otro lado, se utilizaron los resultados del estudio realizado en el ITCM, durante el primer semestre de 2017 (Santiago González et al, 2017), donde se aplicó un cuestionario debidamente validado para identificar el uso de los dispositivos móviles durante su escolaridad en carreras de ingeniería.

El cuestionario se aplicó a 356 estudiantes como una muestra representativa de la matrícula del ITCM, estratificada por carrera, por género $\mathrm{y}$ por semestre de avance, que permitiría identificar las percepciones y los hábitos de los estudiantes en relación con los dispositivos móviles, al encontrarse inscritos en un programa de ingeniería.

Lo anterior con el fin de identificar los usos de los dispositivos móviles por parte de los estudiantes, pero también de sus profesores, e identificar algunos posibles indicios de su correlación con otros indicadores como el género, el semestre cursado, y la carrera que estudian.

Así mismo, se consideraron los resultados del estudio exploratorio sobre las competencias digitales, informacionales y pedagógicas de los profesores, así como sus actitudes hacia las Tic, realizado durante el 2017. 
En dicho estudio se compararon las respuestas de una muestra conveniente de profesores del ITCM y de la Universidad de Salamanca en España. Los instrumentos fueron dos cuestionarios debidamente validados de una propuesta de Rangel Baca (2015) y Tejedor, García-Valcárcel, y Prada (2009), de acuerdo con Saldaña (2017). Los análisis estadísticos se realizaron utilizando hojas de cálculo y el programa SPSS de IBM.

\section{Resultados sobre el EXANI-II}

La población sustentante estuvo constituida por un $65 \%$ de hombres y $35 \%$ de mujeres, de los cuales el $76 \%$ provenía de un bachillerato con régimen de sostenimiento público y el $24 \%$ de una escuela privada. Así también 58.6\% provenía de bachillerato tecnológico y $34.9 \%$ de bachillerato general.

La tabla 1 muestra la distribución de frecuencias de los 1581 sustentantes por carrera: Ingeniería Ambiental (IA), Ingeniería Eléctrica (IE), Ingeniería Electrónica (IEo), Ingeniería en Geociencias (IG), Ingeniería en Gestión Empresarial (IGE), Ingeniería Industrial (II), Ingeniería Mecánica (IM), Ingeniería Petrolera (IP), Ingeniería Química (IQ) e Ingeniería en Sistemas Computacionales (ISC).

\begin{tabular}{|l|r|r|}
\hline Carrera & Sustentantes & Porcentaje \\
\hline IA & 64 & 4 \\
\hline IE & 129 & 8.2 \\
\hline IEo & 84 & 5.3 \\
\hline IG & 137 & 8.7 \\
\hline IGE & 184 & 11.6 \\
\hline II & 278 & 17.6 \\
\hline IM & 219 & 13.9 \\
\hline IP & 96 & 6.1 \\
\hline IQ & 262 & 16.6 \\
\hline ISC & 128 & 8.1 \\
\hline
\end{tabular}

Tabla 1 Distribución de frecuencias de la población sustentante por carrera

Fuente: Elaboración Propia

\section{Algunos resultados del Cuestionario de Contexto del EXANI-II}

El análisis de la confiabilidad de los datos obtenidos para las preguntas sobre el uso del dispositivo móvil -para leer, buscar información o comunicarse con sus compañeros para fines de tareas escolares- se realizó mediante el alfa de Cronbach y resultó ser aceptable -0.774- cuando están asociados a los resultados de sus exámenes de admisión.
En cuanto a las respuestas, la gran mayoría de los aspirantes al ITCM manifestaron que utilizan sus dispositivos móviles como instrumentos para resolver sus tareas (94\%) y para comunicarse con sus compañeros por el mismo asunto (89\%).

Mientras que, apenas un poco más de la tercera parte de los estudiantes dijo que utilizaba su dispositivo móvil para leer periódicos, libros o revistas $(35.8 \%)$. En la tabla 2 se presentan los porcentajes de la población estudiantil que respondió utilizar frecuentemente o casi siempre su dispositivo móvil -celular, tableta o computadora personal- para cada una de las actividades de: leer periódicos, libros o revistas; buscar información para tareas escolares; o comunicarse con sus compañeros por tareas escolares.

\begin{tabular}{|c|c|c|c|c|}
\hline \multirow[b]{2}{*}{ Carrera } & \multirow{2}{*}{ 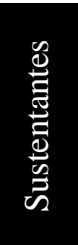 } & \multicolumn{3}{|c|}{$\begin{array}{l}\text { Usa frecuentemente o casi siempre el } \\
\text { dispositivo móvil para: }\end{array}$} \\
\hline & & $\begin{array}{c}\text { leer } \\
\text { periódicos, } \\
\text { libros o } \\
\text { revistas }\end{array}$ & $\begin{array}{c}\text { buscar } \\
\text { información } \\
\text { para tareas }\end{array}$ & $\begin{array}{c}\text { comunicarse } \\
\text { con los } \\
\text { compañeros } \\
\text { por tareas }\end{array}$ \\
\hline IA & 64 & 43.8 & 96.9 & 93.8 \\
\hline IE & 129 & 31.8 & 93.8 & 87.6 \\
\hline IEo & 84 & 31.0 & 91.7 & 84.5 \\
\hline $\mathrm{IG}$ & 137 & 36.5 & 94.2 & 94.2 \\
\hline IGE & 184 & 42.9 & 94.2 & 90.2 \\
\hline II & 278 & 33.1 & 94.6 & 88.8 \\
\hline IM & 219 & 32.9 & 91.8 & 85.9 \\
\hline IP & 96 & 31.3 & 90.6 & 86.5 \\
\hline IQ & 262 & 41.6 & 96.9 & 94.7 \\
\hline ISC & 128 & 32.8 & 95.3 & 82.0 \\
\hline Total & 1581 & 35.8 & 98.0 & 88.8 \\
\hline
\end{tabular}

Tabla 2 Respuestas a preguntas de contexto en el examen EXANI-II Admisión 2016

Fuente: Elaboración Propia

Estos resultados se analizaron de forma estadística para verificar si existían diferencias significativas en el uso de los dispositivos móviles por la modalidad o el régimen de bachillerato de procedencia. Los resultados fueron negativos, al igual que en el caso de cada una de las carreras a las que aspiran.

Esto es, todos los estudiantes se comportan igual con respecto al uso de los dispositivos móviles, no importa si son de una carrera u otra, si provienen de un tipo de bachillerato u otro, o si vienen de una escuela pública o privada. 
Sin embargo, las pruebas de hipótesis, sí encontraron diferencias significativas en el uso de los dispositivos móviles para leer periódicos, libros o revistas, así como para buscar información durante la realización de tareas escolares, de acuerdo con el género del aspirante.

Las pruebas de correlación respectivas permitieron observar una relación positiva entre el uso de los dispositivos móviles para leer periódicos, libros o revistas con el género femenino de las sustentantes (0.112). Es decir, las mujeres utilizan esa tecnología de manera más eficaz en lo escolar, que los varones.

\section{Algunos resultados del EXANI-II Admisión 2016}

Los resultados globales para el examen EXANIII Admisión 2016 -en términos del índice CENEVAL denominado ICNE- obtenidos por los sustentantes y su comparativo con los resultados nacionales se presentan en la tabla 3 . De acuerdo con dicha tabla 3, el promedio de los exámenes de habilidades de Pensamiento Matemático, Pensamiento Analítico, Estructura de la Lengua y Comprensión Lectora muestra que los aspirantes a ingresar al ITCM obtienen mejores resultados que los promedios estatal y nacional.

\begin{tabular}{|l|r|r|r|}
\hline $\begin{array}{c}\text { Modalidad } \\
\text { de } \\
\text { procedencia }\end{array}$ & \multicolumn{1}{|c|}{$\begin{array}{c}\text { Global en } \\
\text { ITCM }\end{array}$} & \multicolumn{1}{c|}{$\begin{array}{c}\text { Global } \\
\text { estatal }\end{array}$} & \multicolumn{1}{c|}{$\begin{array}{c}\text { Global } \\
\text { nacional }\end{array}$} \\
\hline sustentantes & $\begin{array}{l}\text { 24973 } \\
\text { sustentantes }\end{array}$ & $\begin{array}{l}\text { 748562 } \\
\text { sustentantes }\end{array}$ \\
\hline $\begin{array}{l}\text { Promedio } \\
\text { general }\end{array}$ & 1027 & 978 & 999 \\
\hline $\begin{array}{l}\text { Bachillerato } \\
\text { general }\end{array}$ & 1020 & 978 & 1005 \\
\hline $\begin{array}{l}\text { Bachillerato } \\
\text { tecnológico }\end{array}$ & 1031 & 987 & 993 \\
\hline $\begin{array}{l}\text { Profesional } \\
\text { técnico }\end{array}$ & 1018 & 968 & 979 \\
\hline
\end{tabular}

Tabla 3 Resultados globales comparativos del EXANI-II Admisión por modalidad del bachillerato de procedencia del sustentante, en índice ICNE del CENEVAL

Fuente: Elaboración Propia

Aunque los estudiantes sustentantes del examen de admisión provengan de diferentes modalidades de bachillerato, los resultados promedio global son mejores que los promedios de las escuelas del estado y del país. Se realizaron también pruebas de hipótesis con los resultados del módulo de admisión del EXANIII y las respuestas a las preguntas relacionadas con el uso de los dispositivos móviles para los fines indicados en la tabla 2.
Los resultados mostraron que existían diferencias significativas en los resultados de los exámenes de Pensamiento Matemático (PMA), Estructura de la Lengua (ELE) y Comprensión Lectora (CLE) para los fines mencionados en la tabla 2. Sin embargo, en Pensamiento Analítico (PAN) no ocurrió lo mismo con la búsqueda de información para tareas escolares mediante el dispositivo móvil. Los coeficientes de correlación de Spearman se muestran en las tablas 4 y 5 .

\begin{tabular}{|c|c|c|c|}
\hline $\begin{array}{c}\text { Utiliza los } \\
\text { dispositivos } \\
\text { móviles para: }\end{array}$ & $\begin{array}{l}\text { Rho de } \\
\text { Spearman }\end{array}$ & $\begin{array}{l}\text { Pensamiento } \\
\text { Matemático } \\
\text { (PMA) }\end{array}$ & $\begin{array}{l}\text { Pensamiento } \\
\text { Analítico } \\
\text { (PAN) }\end{array}$ \\
\hline \multirow[t]{3}{*}{$\begin{array}{l}\text { leer periódicos, } \\
\text { libros o } \\
\text { revistas }\end{array}$} & $\begin{array}{l}\text { Coeficiente } \\
\text { de } \\
\text { correlación }\end{array}$ & $.071 * *$ & $.067 * *$ \\
\hline & \begin{tabular}{|l|}
$\begin{array}{l}\text { Sig. } \\
\text { (bilateral) }\end{array}$ \\
\end{tabular} & 0.005 & 0.007 \\
\hline & $\mathrm{N}$ & 1581 & 1581 \\
\hline \multirow{3}{*}{$\begin{array}{l}\text { buscar } \\
\text { información } \\
\text { por tareas } \\
\text { escolares }\end{array}$} & $\begin{array}{l}\text { Coeficiente } \\
\text { de } \\
\text { correlación }\end{array}$ & $.076^{* * *}$ & 0.045 \\
\hline & $\begin{array}{l}\text { Sig. } \\
\text { (bilateral) }\end{array}$ & 0.003 & 0.072 \\
\hline & $\mathrm{N}$ & 1581 & 1581 \\
\hline \multirow{3}{*}{$\begin{array}{l}\text { hablar con } \\
\text { compañeros } \\
\text { por tareas } \\
\text { escolares }\end{array}$} & $\begin{array}{l}\text { Coeficiente } \\
\text { de } \\
\text { correlación }\end{array}$ & $.063 *$ & $.067 * *$ \\
\hline & $\begin{array}{l}\text { Sig. } \\
\text { (bilateral) }\end{array}$ & 0.012 & 0.007 \\
\hline & $\mathrm{N}$ & 1581 & 1581 \\
\hline
\end{tabular}

Tabla 4 Coeficiente de correlación entre el uso de los dispositivos móviles y el resultado en PMA y PAN del EXANI-II Admisión 2016.

Fuente: Elaboración Propia

\begin{tabular}{|c|c|c|c|}
\hline $\begin{array}{l}\text { Utiliza los } \\
\text { dispositivos } \\
\text { móviles para }\end{array}$ & $\begin{array}{c}\text { Rho de } \\
\text { Spearman }\end{array}$ & $\begin{array}{l}\text { Estructura } \\
\text { de la lengua } \\
(\text { ELE) }\end{array}$ & $\begin{array}{l}\text { Comprensión } \\
\text { lectora (CLE) }\end{array}$ \\
\hline \multirow[t]{3}{*}{$\begin{array}{l}\text { leer periódicos, } \\
\text { libros o revistas }\end{array}$} & $\begin{array}{l}\text { Coeficiente } \\
\text { de } \\
\text { correlación }\end{array}$ & $.125^{* *}$ & $.141^{* *}$ \\
\hline & $\begin{array}{l}\text { Sig. } \\
\text { (bilateral) }\end{array}$ & .000 & .000 \\
\hline & $\mathrm{N}$ & 1581 & 1581 \\
\hline \multirow{3}{*}{$\begin{array}{l}\text { buscar } \\
\text { información } \\
\text { por tareas } \\
\text { escolares }\end{array}$} & $\begin{array}{l}\text { Coeficiente } \\
\text { de } \\
\text { correlación }\end{array}$ & $.080^{* * *}$ & $.111^{* * *}$ \\
\hline & $\begin{array}{l}\text { Sig. } \\
\text { (bilateral) }\end{array}$ & .001 & .000 \\
\hline & $\mathrm{N}$ & 1581 & 1581 \\
\hline \multirow{3}{*}{$\begin{array}{l}\text { hablar con } \\
\text { compañeros } \\
\text { por tareas } \\
\text { escolares }\end{array}$} & $\begin{array}{l}\text { Coeficiente } \\
\text { de } \\
\text { correlación }\end{array}$ & $.094^{* *}$ & $.096^{* *}$ \\
\hline & $\begin{array}{l}\text { Sig. } \\
\text { (bilateral) }\end{array}$ & .000 & .000 \\
\hline & $\mathrm{N}$ & 1581 & 1581 \\
\hline
\end{tabular}

Tabla 5 Coeficiente de correlación entre el uso de los dispositivos móviles y el resultado en ELE y CLE del EXANI-II Admisión 2016

Fuente: Elaboración Propia

SOTO-HERNÁNDEZ, Ana María, VARGAS-PÉREZ, Laura Silvia PERALTA-ESCOBAR, Jorge y SALDAÑA-GARCÍA, Sergio, Tecnología móvil en el ambiente académico y su uso en videojuegos. Revista de Simulación Computacional. 2019 
De la tabla 4, se deduce que buscar información en el dispositivo móvil para tareas no ayuda a mejorar el resultado de PAN.

\section{Algunos resultados del Módulo de Ingeniería del EXANI-II}

El análisis de correlación sobre el uso de los dispositivos móviles para leer periódicos, libros o revistas, el uso del dispositivo móvil para la búsqueda de información para las tareas escolares y su uso para comunicarse con sus compañeros por cuestiones de los cursos, con cada una de las cuatro áreas del examen de diagnóstico del Módulo de Ingeniería Matemáticas, Física, Lenguaje Escrito e Inglésarrojó los resultados mostrados en la tabla 6 .

\begin{tabular}{|c|c|c|c|c|c|}
\hline $\begin{array}{c}\text { Utiliza los } \\
\text { dispositivos } \\
\text { móviles para: }\end{array}$ & $\begin{array}{c}\text { Rho de } \\
\text { Spearman }\end{array}$ & $\mathbf{M}$ & $\mathbf{F}$ & $\mathbf{L E}$ & I \\
\hline \multirow[t]{3}{*}{$\begin{array}{l}\text { leer periódicos, } \\
\text { libros o revistas }\end{array}$} & $\begin{array}{l}\text { Coeficiente } \\
\text { de correlación }\end{array}$ & 0.015 & 0.043 & $.115^{* *}$ & $.092^{* *}$ \\
\hline & $\begin{array}{l}\text { Sig. } \\
\text { (bilateral) }\end{array}$ & 0.552 & 0.09 & 0 & 0 \\
\hline & $\mathrm{N}$ & 1581 & 1581 & 1581 & 1581 \\
\hline \multirow{3}{*}{$\begin{array}{l}\text { buscar } \\
\text { información para } \\
\text { tareas escolares }\end{array}$} & $\begin{array}{l}\text { Coeficiente } \\
\text { de correlación }\end{array}$ & $.053^{*}$ & 0.035 & $.082^{* *}$ & $.055^{*}$ \\
\hline & $\begin{array}{l}\text { Sig. } \\
\text { (bilateral) }\end{array}$ & 0.034 & 0.16 & 0.001 & 0.029 \\
\hline & $\mathrm{N}$ & 1581 & 1581 & 1581 & 1581 \\
\hline \multirow{3}{*}{$\begin{array}{ll}\text { hablar } & \text { con } \\
\text { compañeros } & \text { por } \\
\text { tareas escolares }\end{array}$} & $\begin{array}{l}\text { Coeficiente } \\
\text { de correlación }\end{array}$ & 0.034 & $0.001^{-}$ & $.099^{* *}$ & $.064^{*}$ \\
\hline & $\begin{array}{l}\text { Sig. } \\
\text { (bilateral) }\end{array}$ & 0.173 & 0.981 & 0 & 0.011 \\
\hline & $\mathrm{N}$ & 1581 & 1581 & 1581 & 1581 \\
\hline
\end{tabular}

** La correlación es significativa en el nivel 0.01 (bilateral).

* La correlación es significativa en el nivel 0.05 (bilateral).

Tabla 6 Correlación del Módulo Ingeniería EXANI-II 2016 con preguntas sobre dispositivos móviles

Fuente: Elaboración Propia

Como se observa, los resultados del examen de Física $(\mathrm{F})$ no están correlacionados con ninguna de las actividades involucradas en el uso de los dispositivos móviles manifestados previamente.

Los resultados de Matemáticas (M) solamente están correlacionados cuando el dispositivo móvil se utiliza para buscar información que ayude en las tareas escolares como pudieran ser los videos en YouTube del Profe Julio para matemáticas, por ejemplo.
En el caso de Lenguaje Escrito (LE) e Inglés (I), ambos están correlacionados con los tres usos de los dispositivos móviles estudiados. Quizá en este caso ayude la lectura para todo fin, por lo que se les pudiera motivar para ello.

\section{Sobre el uso de los dispositivos móviles durante la carrera de ingeniería}

Se realizó un estudio el primer semestre de 2017 mediante un cuestionario, para identificar el tipo de dispositivo móvil utilizado por los estudiantes, el uso dado al mismo, las destrezas en su uso, el tipo de aplicaciones que maneja, y los profesores que lo utilizan en sus cursos (Santiago, Pérez y Soto, 2017). Las preguntas presentaban opción de respuesta en una escala de Likert de 0 a 4.

La muestra estratificada de estudiantes se diseñó por género, por carrera y por semestre en el que se encontraban inscritos. Los estudiantes que contestaron el cuestionario conformaron una población constituida por $65 \%$ hombres y $35 \%$ mujeres. La distribución de la muestra, por carrera, se presenta en la tabla 7.

\begin{tabular}{|c|c|c|}
\hline \multirow{2}{*}{ 胥 } & \multicolumn{2}{|c|}{ Muestra } \\
\hline & Sujetos & $\%$ \\
\hline IA & 20 & $6 \%$ \\
\hline $\mathrm{IE}$ & 31 & $9 \%$ \\
\hline IEo & 23 & $6 \%$ \\
\hline $\mathrm{IG}$ & 48 & $13 \%$ \\
\hline IGE & 35 & $10 \%$ \\
\hline II & 63 & $18 \%$ \\
\hline IM & 37 & $10 \%$ \\
\hline IP & 18 & $5 \%$ \\
\hline IQ & 57 & $16 \%$ \\
\hline ISC & 24 & $7 \%$ \\
\hline Total & 356 & $100 \%$ \\
\hline
\end{tabular}

Tabla 7 Distribución porcentual por carrera de la muestra de estudiantes del ITCM

Fuente: Elaboración Propia

La distribución de la muestra, por semestre de avance en la carrera para cada uno de los estudiantes se puede observar en la tabla 8. 


\begin{tabular}{|l|r|}
\hline Semestre & Muestra \\
\hline $1 \mathrm{o}$ & $9.6 \%$ \\
\hline $2 \mathrm{o}$ & $11.0 \%$ \\
\hline $3 \mathrm{o}$ & $5.1 \%$ \\
\hline $4 \mathrm{o}$ & $17.4 \%$ \\
\hline $5 \mathrm{o}$ & $7.0 \%$ \\
\hline $6 \mathrm{o}$ & $13.8 \%$ \\
\hline $7 \mathrm{o}$ & $5.1 \%$ \\
\hline $8 \mathrm{o}$ & $15.2 \%$ \\
\hline $9 \mathrm{o}$ & $3.4 \%$ \\
\hline $10 \mathrm{o}$ & $8.7 \%$ \\
\hline $11 \mathrm{o}$ & $1.4 \%$ \\
\hline $12 \mathrm{o}$ & $2.5 \%$ \\
\hline & $100.0 \%$ \\
\hline
\end{tabular}

Tabla 8 Distribución porcentual de la muestra por semestre de avance de los estudiantes

Fuente: Elaboración Propia

Los resultados iniciales mostraron que $86 \%$ de los estudiantes tienen un teléfono inteligente, $72 \%$ contaban con servicio de navegación sin necesidad de una conexión gratuita, esto es $28 \%$ de los estudiantes requieren la conexión de Wifi para navegar en internet. Los estudiantes también reportaron que el número de profesores que utiliza en su clase el teléfono inteligente era en promedio 2.0; y solamente el $23 \%$ afirmó que 3 o más profesores de este periodo escolar utilizaban los dispositivos móviles para sus cursos (Santiago, Pérez y Soto, 2017).

La tabla 9 presenta el promedio de uso del dispositivo móvil en los estudiantes y el porcentaje de ellos que lo hacen siempre o casi siempre. El $87 \%$ de los estudiantes reconocía utilizar los dispositivos móviles para mantenerse comunicados a través de las redes sociales y el $68 \%$ utiliza aplicaciones y canales de entretenimiento para el ocio, incluyendo las de videojuegos. Mientras que el $47 \%$ los utiliza para aprender fuera del aula, lo cual puede ser el reflejo del uso de diversos medios que ofrecen oportunidades de respuesta a sus inquietudes académicas, entre ellas la plataforma de Khan Academy con la que trabajan varias escuelas de bachillerato.

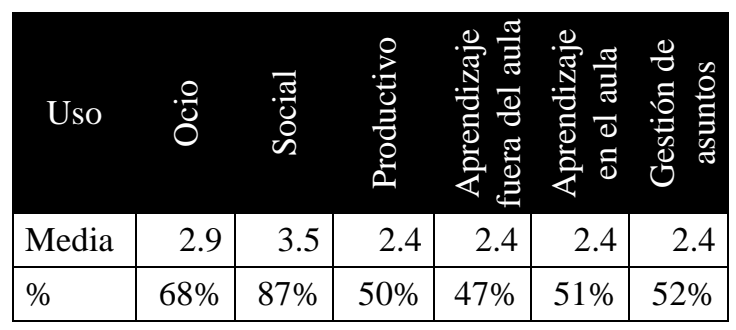

Tabla 9 Uso frecuente de los dispositivos móviles Fuente: Elaboración Propia
Cuando se les preguntó a los estudiantes si conocían y utilizaban diversas aplicaciones, el 93\% afirmó conocer y utilizar redes sociales, el $24 \%$ aplicaciones educativas, y solo el $17 \%$ conoce las plataformas virtuales para la gestión del aprendizaje -Learning Management System, LMS por sus siglas en inglés- como pueden ser Moodle, Schoology, Claroline. Lo anterior seguramente porque sus profesores no las utilizan.

Por otra parte, como se observa en la tabla 10, al preguntarles sobre las aplicaciones de entretenimiento, como los videojuegos, el $51 \%$ afirma utilizarlas.

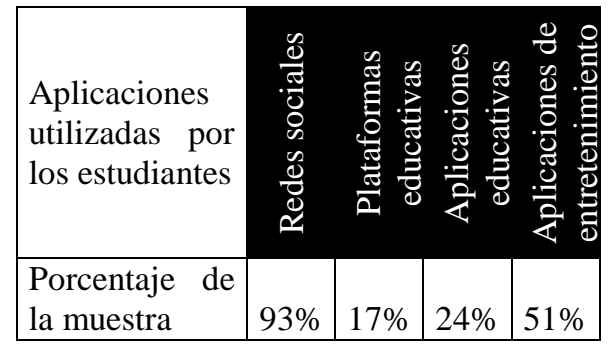

Tabla 10 Aplicaciones utilizadas en el dispositivo móvil Fuente: Elaboración Propia

Se les preguntó también a los estudiantes sobre sus habilidades al utilizar el dispositivo móvil; las respuestas se presentan en la tabla 11 donde se observa el promedio y la desviación estándar para cada habilidad. La media más alta se encuentra en el manejo de la configuración, el uso de la cámara y de las notificaciones.

\begin{tabular}{|l|c|r|r|r|r|}
\hline & N & \multicolumn{2}{c|}{ Mínimo Máximo } & Media & Desv. \\
\hline Gestos & 356 & 0 & 4 & 2.85 & 1.055 \\
\hline Notificaciones & 356 & 0 & 4 & 3.18 & 0.900 \\
\hline Instalaciones & 356 & 0 & 4 & 3.09 & 1.106 \\
\hline Cámara & 356 & 0 & 4 & 3.27 & 0.996 \\
\hline Configuración & 355 & 0 & 4 & 3.33 & 0.909 \\
\hline Nube & 356 & 0 & 4 & 2.45 & 1.355 \\
\hline Sincronizar & 355 & 0 & 4 & 2.76 & 1.242 \\
\hline
\end{tabular}

Tabla 11 Percepción de los estudiantes sobre algunas de sus habilidades con el dispositivo móvil

Fuente: Elaboración Propia

Aunque, es de notar que los valores más altos de desviación se refieren al manejo del espacio virtual de almacenaje $-N u b e$ - la sincronización de dispositivos, y las instalaciones. En general, se observan grandes diferencias en las habilidades para el manejo del dispositivo móvil. 
El análisis estadístico posterior mostró que estas habilidades en particular están asociadas con el género del estudiante masculino- con índices de correlación de 0.132 , 0.139 y 0.178, respectivamente. Estas habilidades no mostraron diferencias significativas entre estudiantes de diferentes carreras, ni de diferentes semestres de avance en las mismas.

Se les preguntó también a los estudiantes sobre las asignaturas en las que trabajaban utilizando este tipo de tecnologías y cuando los dispositivos móviles se hicieran necesarios. Las respuestas se presentan en la tabla 12 , donde se observan diferencias entre la cantidad de profesores de Matemáticas y de ciencias de la ingeniería que utilizan los dispositivos móviles en sus clases, con respecto a otros profesores.

\begin{tabular}{|l|c|}
\hline \multicolumn{1}{|c}{ Área } & $\%$ \\
\hline Matemáticas & $35 \%$ \\
\hline Sociales y administrativas & $13 \%$ \\
\hline Química y Física & $11 \%$ \\
\hline Asignaturas de ciencias de la ingeniería & $35 \%$ \\
\hline Asignaturas de la especialidad & $21 \%$ \\
\hline
\end{tabular}

Tabla 12 Distribución de las asignaturas donde los profesores requieren el uso de un dispositivo móvil Fuente: Elaboración Propia

Aunque se trata solamente de la tercera parte de los estudiantes que han contado con profesores de esas áreas que les incorporan el trabajo con las Tic en los dispositivos móviles, aquellos que se encuentran al frente de las asignaturas de Física, Química, Ciencias Sociales y Administrativas casi no lo hacen. Un porcentaje casi marginal es el que ha sido reportado por los estudiantes.

No se identificó claramente el tipo de uso del celular del profesor con el estudiante, habría que diferenciar si solamente es por medio de las redes sociales, o cuando menos de una plataforma de gestión del aprendizaje.

\section{Sobre el uso de las competencias digitales de los profesores de ingeniería}

El estudio realizado entre la población estudiantil del ITCM -2017- mostró que el 23\% de los estudiantes manifestó que tenían tres o más profesores que utilizaban la tecnología de los dispositivos móviles.
De esos estudiantes, $35 \%$ afirmaron que los profesores de Matemáticas y los de asignaturas de ciencias de la ingeniería eran los que utilizaban dicha tecnología. Los que menos la utilizan son los profesores de Química y Física (11\%) y los de Ciencias Sociales y Administrativas (13\%).

Además, también se ha identificado que los profesores más jóvenes, cuyo manejo de la tecnología estaba presente, no garantizaban una reducción en el índice de reprobación de las asignaturas de los planes de estudio de ingeniería. Los profesores que tenían de 10 a 30 años de antigüedad son los que presentan los mayores índices (Soto Hernández et al, 2015), por lo cual pareciera que el lenguaje tecnológico que utilizan no ha servido para mediar entre estudiante y profesor durante el proceso de aprendizaje.

Posteriormente, en el estudio exploratorio sobre las competencias digitales, informacionales y pedagógicas del profesorado, así como sus actitudes hacia el uso de las TIC, para evaluar la percepción de los profesores del ITCM y de la Universidad de Salamanca en España (Saldaña, 2017), se concluye que los profesores en general tienen una actitud favorable hacia las TIC en su labor docente (más del 70\%), sin embargo también es posible afirmar que la opinión del profesorado no se refleja en sus actos, que más bien pudieran ser más buenas intenciones, aunque muestran su disposición a la capacitación y actualización, de acuerdo con Saldaña (2017).

Dentro de las conclusiones en este estudio de Saldaña (2017) se considera que, en general, "la formación docente no está asociada con el desarrollo de las tecnologías y por consiguiente con la aplicación pedagógica de las mismas para la mejora del aprendizaje" (p. 208).

Lo anterior implica un involucramiento de los liderazgos en las instituciones para conjuntar esfuerzos y mejorar las capacidades docentes, ya que, el exceso desorganizado de información, llamada infoxicación (Innerarity, citado por Saldaña, 2017) debiera enfocarse a través de diversas metodologías que sugiere el propio autor y que son útiles en todos los ámbitos: personales, de investigación, y de docencia. 


\section{Discusión y conclusiones}

El uso de los dispositivos móviles durante el proceso escolarizado se ha generalizado $y$ convertido en una minoría a quienes no poseen un teléfono inteligente, en el sur de Tamaulipas al menos. Esto sí es una situación de exclusión para estos estudiantes y las instituciones educativas, tanto de nivel medio superior como superior, debe proveer los medios para garantizar el acceso a recursos tecnológicos y mediar en esa desventaja. De otra manera el estudiante empleará posiblemente un recurso económico para acudir a un ciber dejando de lado la atención a otras necesidades prioritarias.

\section{Sobre la relación entre habilidades y conocimientos del EXANI-II y los dispositivos móviles}

El uso del dispositivo móvil para leer periódicos, libros o revistas, como ayuda para buscar información para tareas escolares, y para comunicarse con sus compañeros por tareas escolares, se analizó con respecto a los resultados obtenidos en los ocho exámenes presentados en el EXANI-II.

Se resume que para los exámenes de habilidades: utilizar el dispositivo móvil para leer periódicos, libros o revistas, para buscar información como ayuda para tareas escolares y utilizarlo para comunicarse con sus compañeros por asuntos de las mismas tareas, se encuentran correlacionados con sus resultados en Pensamiento Matemático, Estructura de la Lengua y Comprensión Lectora. En el caso del Pensamiento Analítico, existe correlación entre el primero y tercer uso del dispositivo móvil, pero no así en el segundo referido a la búsqueda de la información.

Se resume que para los exámenes de conocimientos: utilizar el dispositivo móvil para leer periódicos, libros o revistas está correlacionado con mejores resultados para los conocimientos de Lenguaje Escrito e Inglés; utilizar el dispositivo móvil para buscar información como ayuda para tareas escolares está correlacionado con mejores resultados para los conocimientos sobre Matemáticas, Lenguaje Escrito, e Inglés; y utilizar el dispositivo móvil para comunicarse con sus compañeros por asuntos de tareas escolares está correlacionado con los conocimientos sobre Lenguaje Escrito, e Inglés. En el caso de Física no se encontró ninguna correlación.
De todo lo anterior se podría decir que el uso de los dispositivos móviles, en cualquiera de los modos estudiados, no está relacionado con buenos resultados en conocimientos sobre Física. Y tampoco lo están los conocimientos de Matemáticas con utilizar el dispositivo móvil para comunicarse con sus compañeros por asuntos escolares. Como tampoco lo está con buenos resultados en Pensamiento Analítico si se busca información para tareas a través del dispositivo móvil.

\section{Sobre el uso de los dispositivos móviles de los estudiantes de ingeniería}

El manejo de los dispositivos móviles por parte de los estudiantes de ingeniería, tanto de primer semestre como de semestres avanzados, se puede decir que no representa conflictos. No importa la carrera que estudian, ni el semestre que cursan.

Así también, puede decirse que las chicas deben fortalecer sus habilidades tecnológicas, sobre todo en el manejo de las instalaciones de aplicaciones, de la Nube, de la sincronización de dispositivos, y del uso de los emoticones en sus dispositivos móviles.

Pero también, puede decirse que ellas tienen mejor disposición para utilizar el dispositivo móvil para leer periódicos, libros o revistas, y para buscar información que les ayude en la realización de sus tareas escolares, que los varones. Como ya se esperaba, las aplicaciones más utilizadas por los estudiantes son las diversas redes sociales. Sin embargo, también se comprobó que el uso de aplicaciones de entretenimiento, como los videojuegos, tienen una preferencia de poco más de la mitad de la población estudiada.

\section{Sobre el uso de las Tic por los profesores}

El uso de las tecnologías de la información y la comunicación por parte de los profesores del ITCM no ha sido una práctica institucional ni sistemática. Los esfuerzos han sido personales y, algunos generados por cuerpos colegiados que no han alcanzado la característica de sistémicos dentro de ese ámbito. Se observan entonces iniciativas aisladas que se pierden en los intentos diversos por desarrollar conjuntamente con los estudiantes un ambiente y espacios de aprendizaje enriquecidos con las Tic. 
Los profesores afirman estar dispuestos y con actitudes positivas ante el uso de las Tic (70\%), incluyendo las necesidades de capacitación y actualización, sin embargo, los estudiantes reportan que solamente un máximo de $35 \%$ de los profesores de algunas áreas son los que trabajan con ellos utilizándolas, y solamente un $23 \%$ de los estudiantes tiene tres o más profesores que les demandan su uso.

Los esfuerzos de las academias de profesores de Matemáticas, y de las asignaturas de las carreras parecieran ir avanzando, aunque no en la medida que dicen que lo hacen (35\% vs $70 \%$ ). El resto realmente se ha quedado rezagado de los avances tecnológicos.

\section{Conclusiones sobre los resultados en el uso de dispositivos móviles}

El uso extendido de los dispositivos móviles ha propiciado una nueva vía para los estudiantes en su proceso de aprendizaje. En particular, para aquellos que aspiran o estudian una carrera de ingeniería en el sur de Tamaulipas. Ya no es necesario traer una computadora portátil ni acudir a un laboratorio de cómputo para tener acceso a la información disponible en internet.

Puede decirse que se encuentra ampliamente utilizado el dispositivo móvil por parte de los estudiantes, aunque se tiene todavía una porción de alrededor del $14 \%$ de la matrícula del ITCM que no tiene acceso a esa tecnología; seguramente por cuestiones económicas derivadas principalmente de los casos de estudiantes foráneos. Garantizar su acceso a ellas debe ser un compromiso institucional para minimizar la inequidad. El servicio del Wifi institucional cerraría esa brecha.

El uso de los dispositivos móviles requiere de diversas competencias que los estudiantes del sur de Tamaulipas adquieren y fortalecen por el ambiente en el que se han desarrollado (García Martín, García-Sánchez, Álvarez-Fernández, y Díez-Caso, 2014).

No obstante, se identificó que las estudiantes se perciben como menos competentes en la instalación de aplicaciones, de la Nube, en la sincronización de dispositivos y en el uso de los emoticones para sus conversaciones personales.
Lo cual quizá sería un área de oportunidad para capacitar a todos los estudiantes en esas operaciones digitales porque al final serán de utilidad no solamente en su vida personal sino en las demandas de sus profesores cuando deben completar un trabajo o tarea escolar.

Sobre las aplicaciones utilizadas por los estudiantes era de esperarse el alto grado en el manejo de las redes sociales (93\%) en los dispositivos móviles, y también el de las aplicaciones de entretenimiento, entre ellos los videojuegos $(51 \%)$.

Lo anterior contrasta con el bajo uso de las aplicaciones (24\%) y las plataformas educativas $(17 \%)$. Lo anterior bien pudiera estar asociado al trabajo de los profesores ya que, aunque se trata de los profesores de ingeniería, se encontró que un máximo de $35 \%$ de los estudiantes indicaron que los profesores de Matemáticas y de las asignaturas de ciencias de la ingeniería trabajan con ellos utilizando aplicaciones asociadas con los dispositivos móviles. El resto de los profesores las utilizan mucho menos.

Esto amerita trabajar en el establecimiento de una estrategia institucional para capacitar y actualizar a los profesores de todos los departamentos en el uso de la tecnología dentro de sus asignaturas, como lo concluyó Saldaña (2017) ya que los estudiantes tienen las competencias pertinentes para ello.

Finalmente, si la tendencia de los jóvenes en esta década es mantenerse conectados y estar interactuando a través de diversos medios, muchas veces virtuales, lúdicos como son los videojuegos, bien pudieran los profesores involucrarse en la búsqueda y el desarrollo de recursos didácticos que motiven a los estudiantes para profundizar en su aprendizaje.

Las metodologías que propician el pensamiento analítico y crítico pudieran tener su primer paso jugando con unas gafas de Realidad Virtual.

\section{Agradecimientos}

Este trabajo es presentado por el Cuerpo Académico ITCMAD-CA-15 del Tecnológico Nacional de México / Instituto Tecnológico de Ciudad Madero. 


\section{Referencias}

Ahern, L., Feller, J., y Nagle, T. (2016). Social media as a support for learning universities: an empirical study of Facebook Groups. Journal of Desicion Systems, 25(51), 35-49. https://doi.org/10.1080/12460125.2016.118742 1

Amry, A. B. (2014). The impact of WhatsApp mobile social learning on the achievement and attitudes of female students. European Scientific Journal 10(22) 116-136.

Bacca, J., Baldiris, S., Fabregat, R., Kinshuk, y Graf, S. (2015). Mobile Augmented Reality in Vocational Education and Training. Procedia. Computer Science, 75, 49-58.

Brandao, D., y Vargas, A. C. (2016). Evaluación del uso de tecnologías digitales en la educación pública. En F. T. Vivo, Experiencias evaluativas de tecnologías digitales en la educación (págs. 9-17). Sao Paulo: Fundación Telefónica Vivo.

Castro Romero, O. (2014). Uso de los medios sociales como herramientas de aprendizaje en educación superior: Análisis comparativo entre México y Corea del Sur. Sinéctica, (44). http://www.sinectica.iteso.mx/articulo/?id=44_ uso_de_los_medios_sociales_

como_herramienta_de_aprendizaje_en_educaci on_superior_analisis_comparativo_

entre_mexico_y_corea_del_sur

CENEVAL. (s.f.). CENEVAL. Recuperado el 25 de Marzo de 2017, de EXANI-II ¿Qué es?: http://www.ceneval.edu.mx/exani-ii\#tab-\$i-9

CENEVAL. (2016). Informe institucional de Resultados del EXANI-II Admisión para el Instituto Tecnológico de Ciudad Madero. México: CENEVAL.

Chen, C. H., Ho, C.-H., y Lin, J.-B. (2015). The development of an augmented reality gamebased learning environment. Procedia Social and Behavioral Sciences, 174, 216-220.

Chiappe, A. (Julio de 2016). Tendencias sobre contenidos educativos digitales en América Latina. (UNESCO/IIPE-OEI, Ed.) Recuperado el 11 de Septiembre de 2016, de Sistema de Información de Tendencias Educativas en América Latina: http://www.siteal.iipe-oei.org
Dahlstrom, E. (2015). Educational Technology and Faculty Development in Higher Education. Louisville: ECAR. www.educause.edu/ecar

Diaz, C., Hincapié, M., y Moreno, G. (2015). How the Type of Content in Educative Augmented Reality Application Affects the Learning Experience. Procedia Computer Science, 75, 205-212.

Ericsson. (2017). Ericsson Mobility Report 2017. Estocolmo: Ericsson.

García Martín, J., García-Sánchez, J. N., Álvarez-Fernández, M. L., y Díez-Caso, H. (2014). Efectos en la competencia digital tras la aplicación de un programa de competencias ocupacionales. European Journal of Education and Psychology, 7(2), 73-81.

Gavilan, D., Martínez-Navarro, G., y FernándezLores, S. (2017). Universitarios y redes sociales informativas: Excépticos totales, moderados duales o pro-digitales. Comunicar, XXV(53), 6170. DOI: https://doi.org/10.3916/C53-2017-06

Gómez-del-Castillo, M. T. (2017). Utilización de WhatsApp para la comunicación en titulados superiores. Revista Iberoamericana sobre Calidad, Eficacia y Cambio en Educación, 15(4), 51-65. DOI: https://doi.org/10.15366/reice2017.15.4.003

Hershkovizt, A., y Forkosh-Baruch, A. (2017). La relación profesor-alumno y la comunicación en Facebook: percepciones de los alumnos. Comunicar(53), 91-101. DOI: https://doi.org/10.3916/C53-2017-09

Hobbs, R., y Tuzel, S. (2017). Teacher motivations for digital and media literacy: An examination of Turkish educators. British Jornal of Educational Technology, 48(1), 7-22. DOI: https://doi.org/10.1111/bjet.12326

Nincarean, D., Ali, M. B., Halim, N. D., y Rahman, M. H. (2013). Mobile Augmented Reality: the potential for education. Procedia. Social and Behavioral Sciences(103), 657-664.

OECD. (2015). How Computers are Related to Students' Performance. En OECD, Students, Computers and Learning: Making the Connection (pp. 145-164). París: OECD Publishing. 
OECD. (2016). México. Nota país. Resultados de PISA 2015. París: OECD.

OECD. (2017). OECD Skills Strategy Diagnostic Report: Mexico. Paris: OECD.

Padilla Zea, N., Collazos Ordoñez, C. A., Gutiérrez Vela, F. L., y Medina Medina, N. (2012). Videojuegos educativos: teorías y propuestas para el aprendizaje en grupo. Ciencia e Ingeniería Neogranadina, 22(1), 139-150.

Pedró, F. (2016). Educación, tecnología y evaluación: hacia un uso pedagógico efectivo de la tecnología en el aula. En F. T. Vivo, Experiencias Evaluativas de Tecnologías Digitales en la Educación (pp. 21-36).Sao Paulo: Fundación Telefónica Vivo.

Rangel Baca, A. (2015). Competencias docentes digitales: Propuesta de un perfil. Pixel-Bit(46), 235-248. DOI: https://doi.org/10.12795/pixelbit.2015.i46.15

Rodríguez Correa, M., y Rivadulla López, J. C. (Julio de 2015). La integración de plataformas de e-learning en la docencia universitaria: percepciones de un grupo de estudiantes sobre los usos de la plataforma Moodle. Revista Electrónica de Investigación y Docencia(14), 27-46.

Saldaña García, S. (2017). Análisis y evaluación de las competencias informacionales y digitales del profesorado de enseñanza superior. En A. M. Soto Hernández, R. G. Camero Berrones, M. B. Sierra Ruiz, C. A. Moreno Mendoza, V. Reyes Méndez, A. Hernández Ramírez, . . . L. S. Vargas Pérez, Formación de ingenieros. Análisis sobre la problemática del aprendizaje del estudiante (pp. 198-210). Puebla: Mariángel.

Samaniego Ocampo, R., y Sarango Salazar, E. (2016). Aplicación de juegos digitales en educación superior. Revista San Gregorio, l(11), 82-91.

Sannikov, S., Zhdanov, F., Chebotarev, P., y Rabinovich, P. (2015). Interactive Educational Content Based on Augmented Reality and 3D Visualization. Procedia Computer Science, 66, 720-729.
Santiago González, N. G., Pérez Hernández, K. I., y Soto Hernández, A. M. (2017). Uso del dispositivo móvil en los estudiantes del ITCM. XXIX Encuentro Nacional de Investigación Científica y Tecnológica del Golfo de México (págs. 22-22). Tampico: ATICTAC.

Soto Hernández, A. M., Ríos Barceló, J. L., Reyes Méndez, V., y Maldonado Soto, O. G. (2015). Los nuevos estudiantes, los viejos profesores, y las Tic's en los cursos de ciencias en ingeniería. En A. M. Soto Hernández, y M. E. De Luna Rodríguez, Las Tic's imperando en las estrategias para el aprendizaje (pp. 221-230). Puebla: Mariángel.

Tejedor, F. J., García-Valcárcel, A., y Prada, S. (2009). Medida de actitudes del profesorado universitario hacia la integración de las TIC. Comunicar, $\quad$ XVII(33), 115-124. DOI: https://doi.org/10.3916/C33.2009.03.00 